\title{
Numerical and Experimental Study of a Flow past a Submerged Mountain Ridge in Shallow Water
}

\author{
by Young-Gill Lee*, Member \\ Makoto Kanai**, Member
}

\author{
Hideaki Miyata**, Member
}

Summary

A numerical computation method for the simulation of the flow around a submerged body of complex geometry is developed in the framework of the TUMMAC (Tokyo Univ. Modified Marker And Cell) method. For the three-dimentional problem, the idea of porosity is introduced in order to simplify the treatment of no-slip body boundary conditions. A flow past a submerged mountain ridge is simulated by the present method in shallow water condition. It is experimentally studied in ship model basin and the results are compared with the computed flow patterns. The complicated interaction of eddies behind a submerged mountain ridge is well simulated.

\section{Introduction}

The analysis of the flow and wave-making phenomena around a physical obstacle is one of the most important subject in the fields of naval architecture, ocean and coastal engineerings. The flows around the bodies of offshore structure or the projecting parts of seabottom are accompanied by complicated vortical motions. Especially, these are more complicated phenomena in the vicinity of free-surface waves. Potential and boundary layer theories have been used to analyze the general features of the flow pattern around a smooth hull surface or a simplified surface of an offshore structure. However, the flows after separation and the nonlinear free-surface have been very grossly treated, because these have very complicated features and the classical theories could not deal with these problems. Therefore, the flow characteristics on complex offshore structures have been mostly treated with experimental methods.

Yamada and Miyata ${ }^{1)}$ suggested the TUMMAC-VII method using a fixed rectangular coordinate system that is applicable to simulate the viscous flow around a three -dimensional complicated body. They treated approximately no-slip body surface condition in the body boundary cells making use of two kinds of porosity

* Department of Naval Architecture and Ocean Engineering, Inha University, Korea

** Department of Naval Architecture and Ocean Engineering, The University of Tokyo

Received 8th July 1992

Read at the Autumn meeting 9, 10th Nov. 1992 parameters and demonstrated by the simulation of a flow past a sphere at the Reynolds number 1000. The complex three-dimensional vortex shedding motions were qualitatively well simulated. Most of the numerical computation methods employ the boundary-fitted coordinate system. However, it will be impossible to generate a useful curvilinear grid system for the boundary surface of complex configuration such as an offshore structure piercing the free-surface which is accompanied with violent nonlinear waves. In case of two-dimensional body, Lee and Miyata had presented a finite-difference method (TUMMAC- $V_{w v}$ method) ${ }^{2), 3}$ using an irregular leg-length in order to treat the noslip body boundary condition in the framework of a rectangular grid system. However, the technique can not be applied to the three-dimensional case, because the program code is extremely complicated and the computation time is remarkably increased. Therefore, the idea of porosity is introduced in order to simplify the treatment of the three-dimensional body boundary condition. Moreover, the treatment of the free-surface conditions, which is used in the TUMMAC-IV method ${ }^{4)}$, is incorporated in the computation of free-surface.

The newly-developed method is applied to a flow around a submerged mountain ridge in shallow water. For the research of this sort, we can cite a good number of papers. Houghton and Kasahara ${ }^{5)}$ investigated the nature of the hydraulic jumps in the flows across a two -dimensional ridge. They used the one-dimensional time-dependent "shallow water" equations for the motion of an incompressible, homogeneous, inviscid and hydrostatic fluid. The nonlinear phenomenon of the jumps was determined by using asymptotic solutions to the model equations. Lamb and Britter ${ }^{6)}$ performed a series of numerical and laboratory experiments for the 
flow over a three-dimensional obstacle in shallow water condition. A finite-difference model was employed in the numerical simulation of a homogeneous non-rotating flow. The results demonstrated how the flow over a three-dimensional obstacle deviates from the flow patterns established for a two-dimensional ridge. Miyata, Matsukawa and Kajitani ${ }^{7)}$ investigated the two-dimensional shallow water flow over an isolated obstacle by a numerical technique based on a finite-difference method (TUMMAC-V method). The computed results indicat ed that the viscous and free-surface flow in a shallow water condition cannot be explained without the understanding of wave breaking nature. Recently, a model for describing the discontinuous atmospheric flows over a mountain ridge was suggested by Cullen ${ }^{8}$. An implicit finite-difference method using Lagrangian evolution equations was presented for modelling such flows.

In this paper, the numerical computation method is described in Section 2. Especially, the treatment of noslip body boundary condition is emphasized in this section. The numerical simulation and experiment for the flow around a shallowly submerged mountain ridge are shown in Section 3. Also, discussions and brief concluding remarks are presented in Section 4.

\section{Numerical computation method}

\subsection{Governing equations}

The governing equations are the Navier-Stokes $(\mathrm{N}-$ $\mathrm{S}$ ) equations and the continuity equation in the case of a three-dimensional incompressible fluid, and represented as follows.

$$
\begin{aligned}
\frac{\partial u_{i}}{\partial t}= & -\frac{\partial \phi}{\partial x_{i}}-\frac{\partial \phi_{t}}{\partial x_{i}}-u_{j} \frac{\partial u_{i}}{\partial x_{j}}+\nu \frac{\partial}{\partial x_{j}}\left(\frac{\partial u_{i}}{\partial x_{j}}\right) \\
& +\nu_{s} \frac{\partial}{\partial x_{j}}\left(\frac{\partial u_{i}}{\partial x_{j}}\right)+\frac{\partial \nu_{s}}{\partial x_{j}}\left(\frac{\partial u_{i}}{\partial x_{j}}+\frac{\partial u_{j}}{\partial x_{i}}\right) \\
& +M_{i}+f_{i}, \\
\frac{\partial u_{j}}{\partial x_{j}}= & 0
\end{aligned}
$$

where $u$ is the velocity component in each direction, $\phi$ $=P / \rho(P$; pressure, $\rho$; density $), \phi_{t}$ is the turbulent pressure per unit density, $\nu$ is the kinematic viscosity and $\nu_{s}$ is the eddy viscosity coefficient from the SGS (Sub-Grid Scale) turbulence model. Also, $M_{i}$ is the arbitrary numerical diffusion, dispersion or dissipation term for the stability of solution, and $f_{i}$ is the external force per unit mass that is the gravitational acceleration in the case of vertical direction component.

\subsection{Grid system}

The computational domain is discretized into a rectangular staggered mesh system. The staggered mesh system lead to the increase of the computational accuracy of mass and momentum conservation property in comparison to a regular mesh system. Since the grid lines do not coincide with the body boundary and freesurface, it can not be anticipated that the computational accuracy is good for the treatment of the body boundary and free-surface conditions. Also, the resolution of the flow simulation in not so high in the vicinity of the boundaries. However, an advantage of this grid system is that the required effort for the grid generation is negligibly small, which is most important for the practical applications.

In order to increase the computational accuracy, the body boundary and free-surface conditions are implemented by several ingenious numerical techniques. That is, a generalized 2nd-order differential scheme and velocity-pressure simultaneous iterative method are used for the body boundary condition, an irregular star and the iterative calculation of marker point are used for the free-surface condition, respectively. Moreover, the computational domain is divided into two regions for the increase of the computational efficiency. The two regions are the fluid region composed of fluid cells ( $\mathrm{F}$-cells) and the body boundary region composed of body boundary cells ( $\mathrm{B}$-cells). The details of the grid system are explained in references (1) and (9).

\section{3 Boundary conditions}

All cells used in computational domain are classified into full-of-fluid cells (F-cells), body boundary cells (B -cells) and empty cells (E-cells). The configuration of a three-dimensional body is represented by two kinds of porosity in B-cells, that is, volume-porosity $\gamma$ and surface-porosity $\beta$ as shown in Fig. 1. the volumeporosity is the ratio of fluid portion in each cell and it is defined at a pressure point. The surface-porosity is the ratio of fluid portion on each surface of a cell as expressed by equation ( 3 ) and it is defined at a veloc. ity point.

$$
\beta_{i+\frac{1}{2}}=\left(\gamma_{i}+\gamma_{i+1}\right) / 2 \text {. }
$$

Moreover, the $\mathrm{B}$-cell is flagged as a cell of which volume-porosity is greater than 0.5 but less than 1.0. A cell of which volume-porosity is less than 0.5 is flagged as E-cell. Also, a F-cell facing a E-cell is defined as a special B-cell ( $\mathrm{B}^{*}$-cell in Fig. 2), because this cell can not be neighboured with six pressure points. Velocity point is assumed to exist when the surface-porosity is
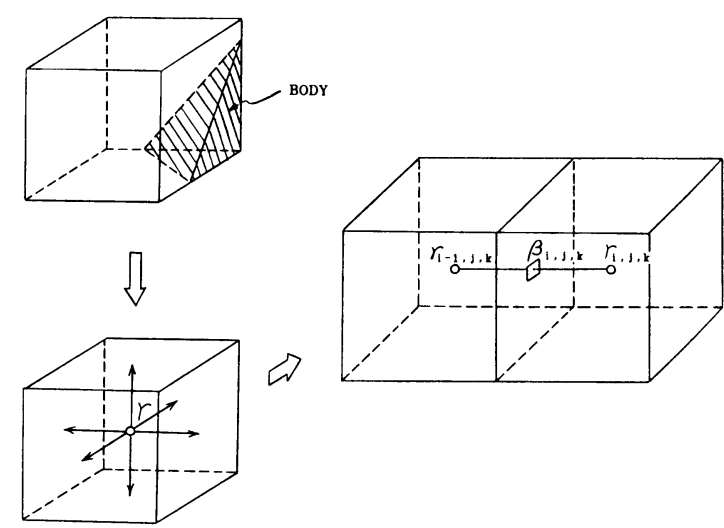

Fig. 1 Definition sketch of volume- and surface-porosity 


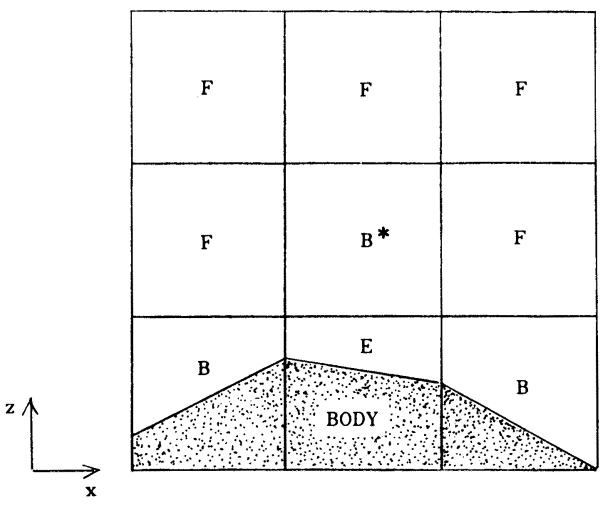

Fig. 2 Cell flagging in TUMMAC-VII scheme.

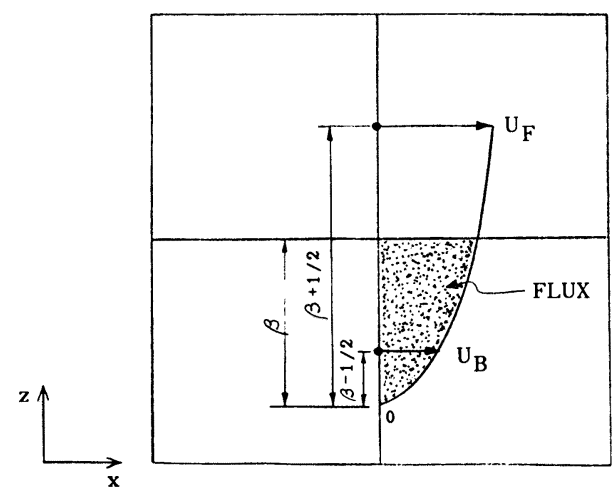

Fig. 3 Interpolation of velocity and definition of flux at a body boundary cell in TUMMAC-VII scheme.

greater than 0.5 . Since the porosity is a scalar value, it is used not only for the flagging of cells and for the calculation of flux and divergence but also for the choice of differencing scheme and for the calculation of first and second derivative terms in the governing equations.

For the no-slip body boundary condition, the velocity in a B-cell, of which surface-porosity is greater than 0.5 but less than 1.0 , is interpolated by the neighbouring velocities and using the definition of zero-velocity on the body surface as shown in Fig. 3. The velocities which are not interpolated themselves are used for the interpolation and the surface-porosity of a cell where neighbouring velocities are situated is used as a weighting factor in the interpolation by a quadratic equation. Also, the no-slip condition is incorporated in the differencing scheme. In the vicinity of body surface, the modified second-order centered differencing is used for the convection and diffusion terms considering the surface-porosity and the zero-velocity on the body surface.

For the calculation of pressure in B-cells the following simultaneous iterative method is used, in order to suitably implement the zero-divergence condition in $\mathrm{B}^{-}$ cells.

$$
\begin{aligned}
\phi^{m+1}= & \phi^{m}+\frac{\omega}{A A} \cdot D, \\
A A= & \Delta t \cdot\left\{\frac{\gamma_{i+1}+\gamma_{i-1}}{(\Delta x)^{2}}+\frac{\gamma_{j+1}+\gamma_{j-1}}{(\Delta y)^{2}}\right. \\
& \left.+\frac{\gamma_{k+1}+\gamma_{k-1}}{(\Delta z)^{2}}\right\},
\end{aligned}
$$

where $D$ is the divergence of a cell, $m$ is the iteration number and $\omega$ is the relaxation factor.

In the free-surface calculation, the exactly same technique of the TUMMAC-IV method ${ }^{4}$ is employed. Namely, the Lagrangian movement of marker particles is used for the fulfilment of the kinematic condition on the free-surface, and the "irregular star" technique of Chan and Street ${ }^{10)}$ is applied to the dynamic condition on that surface. Some interpolation and extrapolation techniques are employed for the determination of the velocity components at the position of a marker particle, i.e., four-point and nine-point interpolations and zero-gradient extrapolations are used.

At the inflow boundary both velocity and pressure distribution are set as the pre-determined values for a uniform flow. In the case of other boundaries the velocities and pressures are set equal to the inner values so that their gradients in the direction normal to the boundary are set zero, that is, a Neumann condition is employed.

\subsection{Computational procedure}

The computational algorithm of the TUMMAC-VII method are well explained in reference (1). Therefore, very brief explanations are described here. The governing equations ( 1 ) and ( 2 ) are represented in finitedifference forms, and solved as an initial-and boundary -value problems including the free-surface conditions using time-marching procedure and iteration methods. The N-S equations are represented by first-order forward differencing in time and second-order centered differencing in a Cartesian coordinate system except for the convection terms. The differencing of the convection terms are described by a third-order upwind differencing scheme. The computation is started from a rested state, and the velocities in computational domain are gradually accelerated for a desired inflow velocity. After the steps of acceleration the computation is continued for an adequate time steps.

The momentum equations and continuity equation result in the Poisson equation for the pressure distribution. Updating of the velocity field is made after updating the pressure field by solving the Poisson equation and this cycle is repeated in the time-marching procedure. The velocity field is updated as the following equation.

$$
\begin{aligned}
u_{i}^{n+1}= & u_{i}^{n}+\Delta t \cdot\left(-\frac{\partial \phi}{\partial x_{i}}-\frac{\partial \phi_{t}}{\partial x_{i}}-C_{i}+D_{i}\right. \\
& \left.+E_{i}+M_{i}+f_{i}\right)^{n},
\end{aligned}
$$

where $\mathrm{n}$ is the number of time step, $C_{i}$ is the convection term, $D_{i}$ is the diffusion term and $E_{i}$ is the eddy viscous 


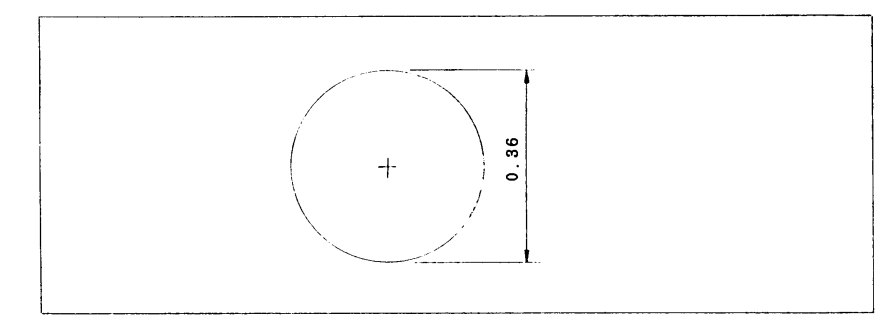

UNITS : m
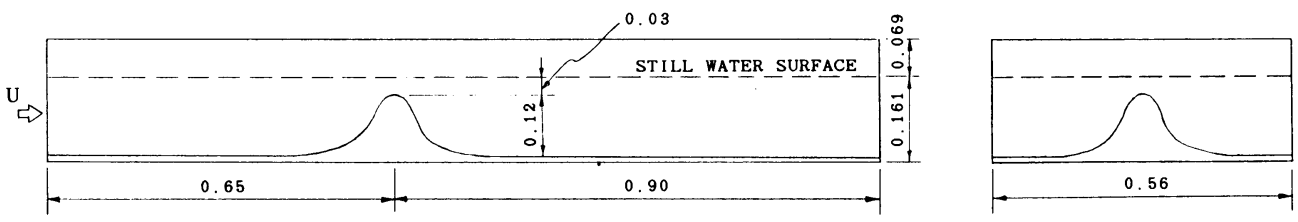

Fig. 4 Computational domain for a mountain ridge in shallow water condition

term. For the determination of the $\phi_{t}$ and $E_{i}$ term, the SGS turbulence model ${ }^{11)}$ is incorporated into the present method so that a complicated flow in high Reynolds number may be well simulated.

For the solution of the Poisson equation the following SOR (Successive Over Relaxation) method is used in F -cells.

$$
\phi_{i, j, k}^{m+1}=\phi_{i, j, k}^{m}+\omega\left(\phi_{i, j, k \text { cal. }}^{m+1}-\phi_{i, j, k}^{m}\right),
$$

where $\phi_{i, j, k c a l}^{m+1}$ is a temporary pressure at each iteration step. The iteration is continued until the second term of Eq. ( 7 ) converges within an allowable error.

\section{Flow pattern around a submerged mountain ridge}

\section{1 Numerical simulation}

The application potentiality of the newly-developed numerical simulation method is examined by simulating the flow pattern around a submerged mountain ridge in shallow water condition. As shown in Fig. 4 the computation was performed about a three-dimensional projecting model of cone type with bottom diameter $0.36 \mathrm{~m}$. The uniform flow which has the velocity $U=0.2 \mathrm{~m} / \mathrm{sec}$ and an empirically determined velocity profile near the bottom surface are provided on inlet surface. Therefore, the Froude number based on the water depth is 0.165 and the Reynolds number based on the height of the projecting part is 23,230. Any other non-dimensional value is based on the height of the projecting part in this paper. As clarified by Lamb and Britter ${ }^{6}$, a hydraulic jump on the free-surface will not appear in this condition.

The grid system in the vicinity of the projecting part is shown in Fig. 5. Since a cell size is uniformly $1 \mathrm{~cm} \times$ $1 \mathrm{~cm} \times 1 \mathrm{~cm}$ all over the computational domain, the height of the projecting part is divided into 12 cells and the total number of cells is 139,000 .

The computation is started from the rest condition,

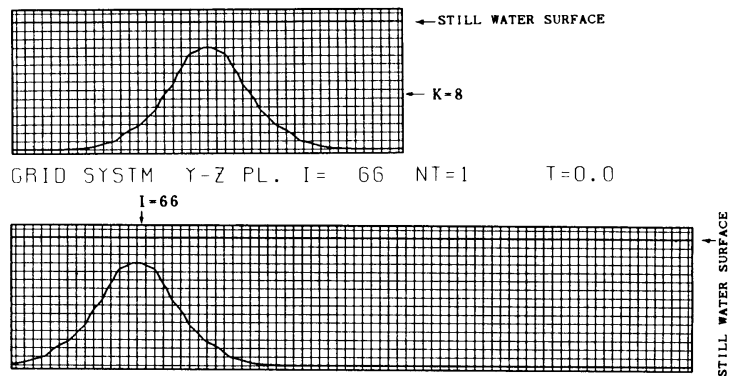

GRID SYSTM $X-Z$ PL. $J=28 \quad N T=1 \quad T=0.0$

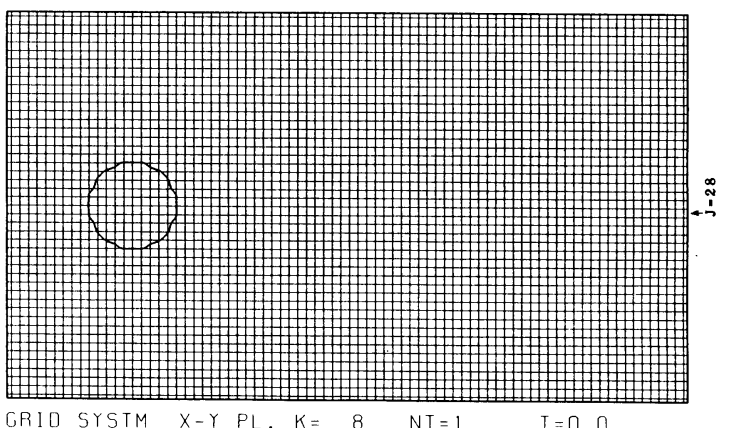

Fig. 5 Grid system in the vicinity of a mountain ridge in shallow water condition.

and the fluid all over the computational domain is accelerated for 1,000 time steps. After the acceleration is finished, the computation is continued to 3,000 time step ( $T=25.0$, where $T$ is the non-dimensional time).

The simulated results are shown in Figs. 6 to 12. In these figures, plane numbers $I, J$ and $K$ are same as observed in Fig. 5. The time-sequential development of the contour maps of vorticity is shown in Figs. 6, 7 and 8 for the cases of the $Y-Z, X-Z$ and $X-Y$ plane, 


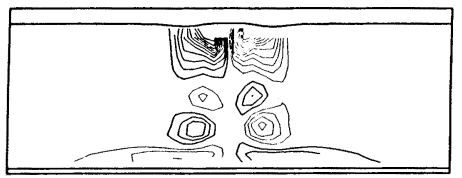

01 CONTOUR $Y-Z$ PL. I $=102 \quad N T=2000 \quad T=16.7$

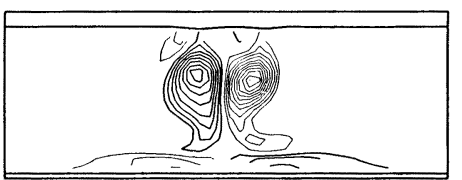

01 CONTOUR $Y-Z$ PL. $I=102 \mathrm{NT}=2250 \quad \mathrm{~T}=18.7$

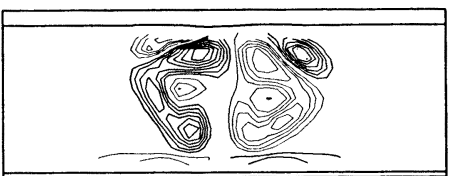

01 CONTOUR $Y-Z$ PL. I $=102 \quad N T=2500 \quad T=20.8$

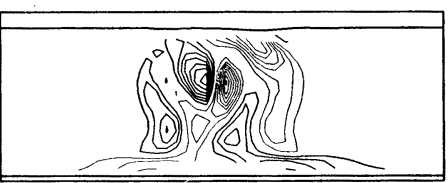

01 CONTOUR $Y-Z$ PL. $I=102 N T=2750 \quad T=22.9$

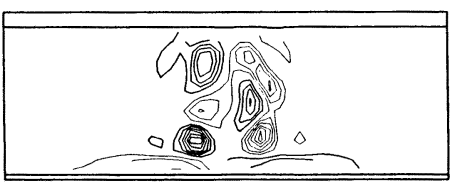

01 CONTOUR $Y-Z$ PL. $I=102 \quad N T=3000 \quad T=25.0$

Fig. 6 Vorticity contour maps on the $Y-Z$ plane at $I=$ 102. Anticlockwise vorticity is contoured in bold lines and the contour interval is $1 / s$.

respectively. The strength of vorticity is calculated by integrating the two-dimensional circulation of a cell and dividing it by the area of the each side surface of a cell. In these figures, $O_{1}$ is the vorticity strength of the rotation about the $x$-axis and $O_{2}, O_{3}$ are those about the $y$-and $z$-axis, respectively. The contour pattern of $O_{1}$ is periodically repeated as observed in Fig. 6 and a large pair of vortices will be recurrently developed as shown at $T=18.7$ is this figure. As shown in Fig. 7, shear flows are developed beneath the free-surface and above the bottom. Also, the vortices generated between the freesurface and the top of the mountain ridge are observed in this figure. Fig. 8 shows the influence of bottom in the shedding motion of vortex component $\mathrm{O}_{3}$. That is, it is clearly observed that the breadth of the wake flow region on the $X-Y$ plane at $K=5$ is narrower than that on te plane at $K=11$.

The simulated wave contour maps are shown in Fig. 9. An intensive non-linear waves are developed just behind the shear flow layer beneath the free-surface. Fig. 10 shows the pressure contour map of each $X-Y$ plane when $T=25.0$. It is found that the intensive non-

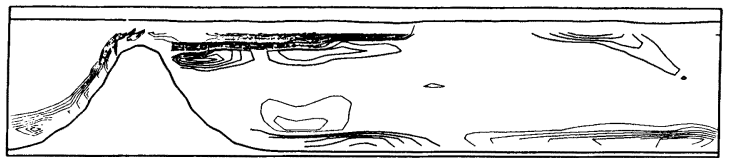

02 CONTOUR $X-Z$ PL. $J=28 \quad N T=2000 \quad T=16.7$

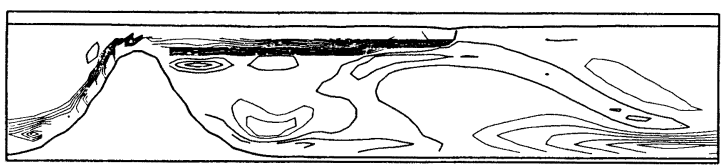

02 CONTOUR $X-Z$ PL. $J=28 \quad N T=2250 \quad T=18.7$

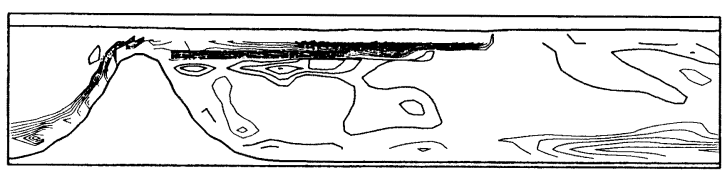

02 CONTOUR $X-Z$ PL. $J=28 \quad N T=2500 \quad T=20.8$

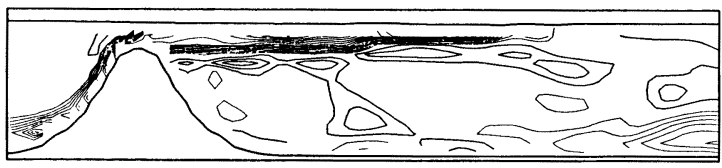

02 CONTOUR $X-Z$ PL. $J=28 \quad N T=2750 \quad T=22.9$

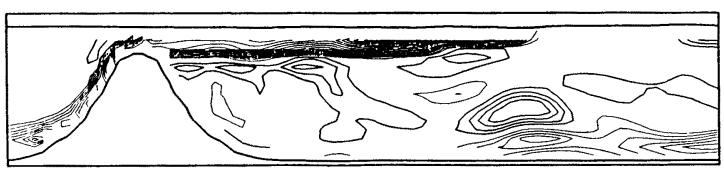

02 CONTOUR $X-Z$ PL. $J=28 \quad N T=3000 \quad T=25.0$

Fig. 7 Vorticity contour maps on the $X-Z$ plane at $J$ $=28$. Anticlockwise vorticity is contoured in bold lines and the contour interval is $1 / s$.

linear waves are not dependent upon the pressure distribution of the $X-Y$ planes below the free-surface. This may be presumably due to a locally strong non-linear interaction between the vortices near the free-surface.

Figs. 11 and 12 show the pressure distribution along the model surface at the $X-Y$ sections and a $X-Z$ section, respectively. Although the grid spacing is not sufficiently fine, the pressure distributions on the model surface are not so fluctuated except for the regions of flow separation.

\section{2 Experiment in towing tank}

For the qualitative validation of the results of the numerical simulation, some flow visualization tests were performed at the ship model basin of the University of Tokyo, which is $86 \mathrm{~m}$ long, $3.5 \mathrm{~m}$ wide and $2.5 \mathrm{~m}$ deep. An axisymmetric isolated mountain ridge which has the same principal dimensions and offsets as the condition of numerical simulation was set in a channel made of two parallel side-plates and one horizontal bottom-plate as shown in Fig. 13. Moreover, in order to stimulate the growth of the boundary layer and turbulent flow in the approach run, a steel mesh $(2.5 \mathrm{~mm} \times 2$. $5 \mathrm{~mm}$ ) of height $10 \mathrm{~mm}$ is placed at the front of the bottom-plate.

In order to perform flow visualization, a slit beam of 

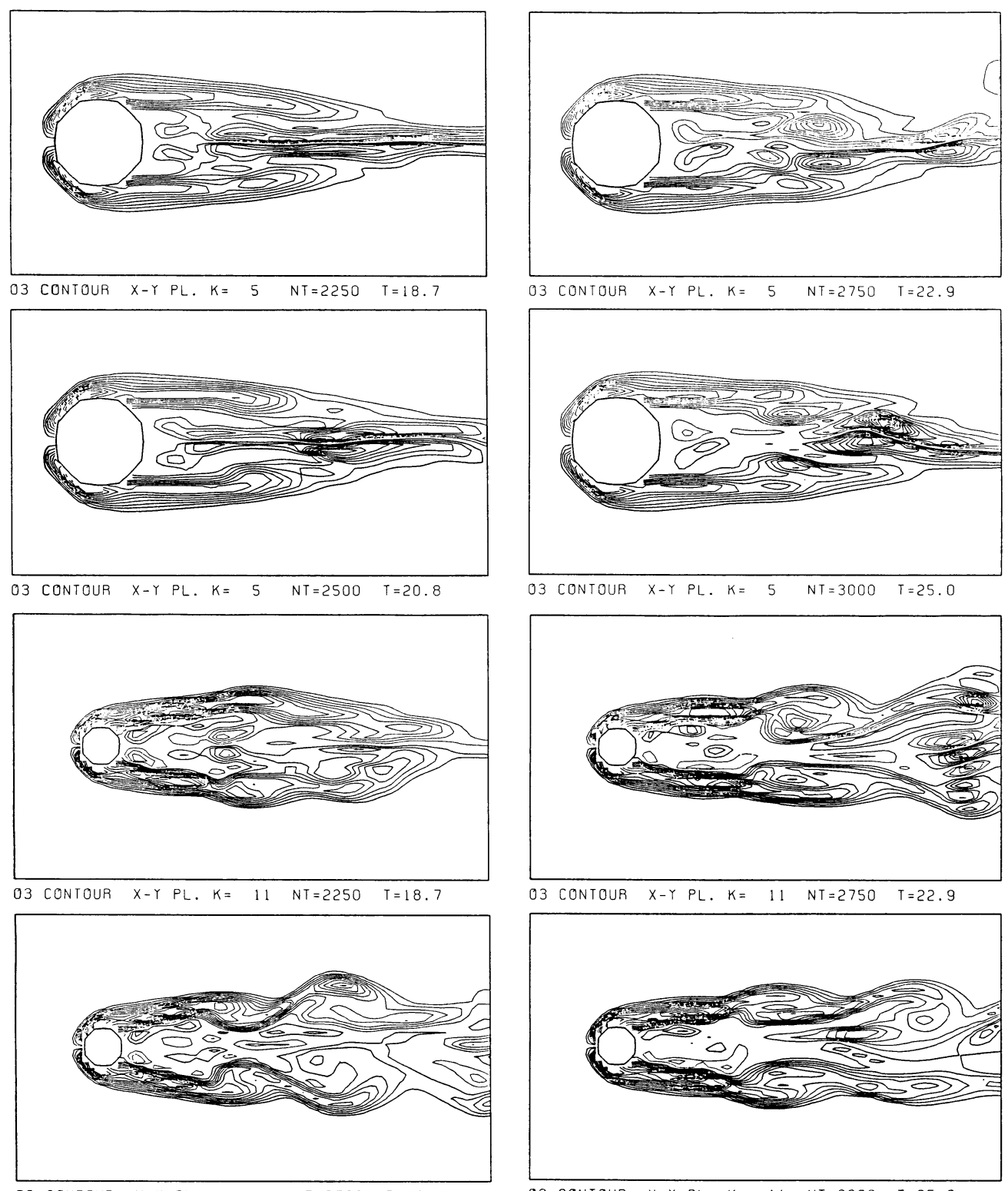

03 CONTOUR $X-Y$ PL. $K=11 \quad N T=2500 \quad T=20.8$

03 CONTOUR $X-Y P L . K=11 \quad N T=3000 \quad T=25.0$

Fig. 8 Vorticity contour maps on the $X-Y$ planes at $K$ $=5$ and $K=11$. Anticlockwise vorticity is contoured in bold lines and the contour interval is $1 / s$. 

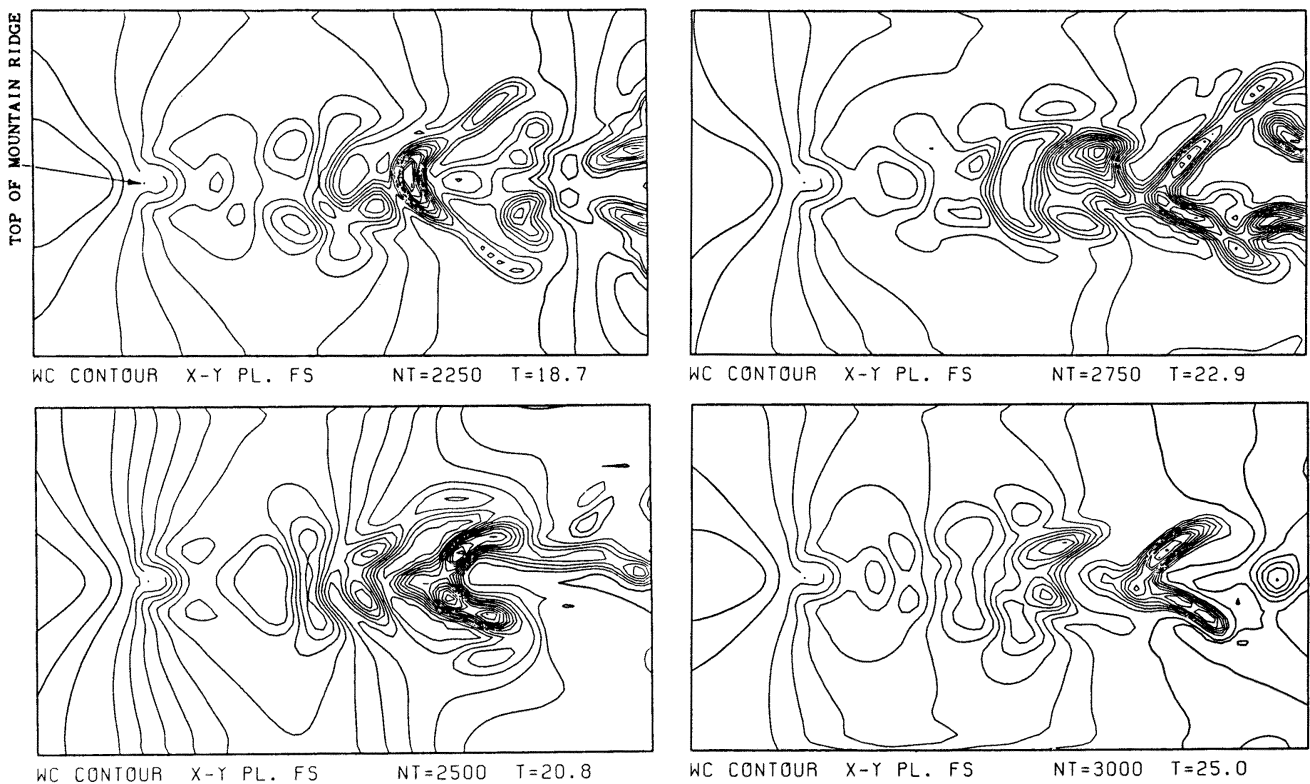

Fig. 9 Wave contour maps above a submerged mountain ridge. Positive wave height is drawn in bold lines and the contour interval is $0.25 \mathrm{~mm}$.
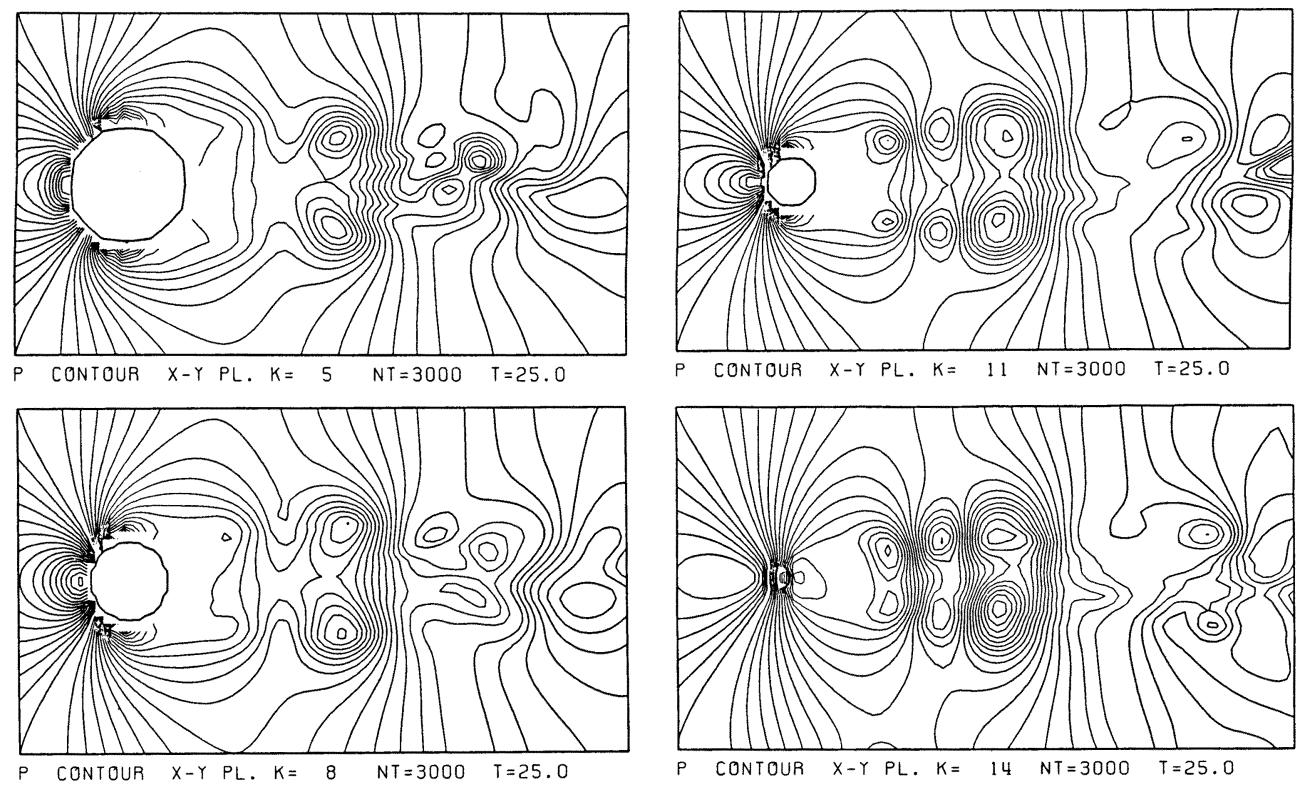

Fig. 10 Pressure contour maps around a submerged mountain ridge at $T=25.0$. Positive pressure coefficient is drawn in bold lines and the contour interval is 0.05 . 


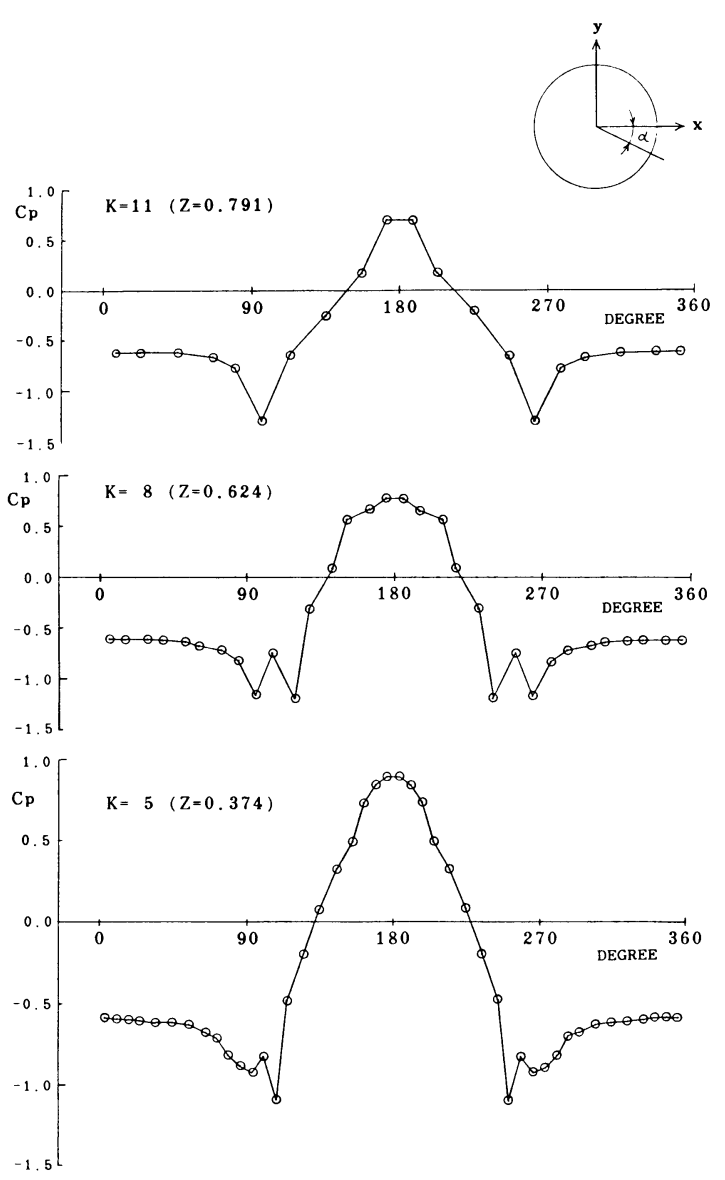

Fig. 11 Surface pressure distribution of a submerged mountain ridge on the $X-Y$ sections at $K=5$, 8 and $11, T=25.0$.

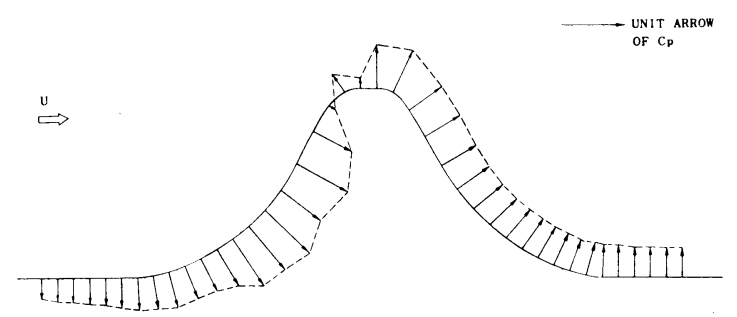

Fig. 12 Surface pressure distribution of a submerged mountain ridge on the $X-Z$ section at $Y=0.0$, $T=25.0$.

light is prepared by a slide projector and photographs are taken by a $35 \mathrm{~mm}$ non-watertight camera. Also, a mirror system is used for the refraction of a slit beam or the setting of the camera. Polystyrene beads coated by a fluorescent material, which are $1.0 \mathrm{~mm}$ in diameter and 1.1 in specific density, are used as tracers. The thickness of the slit beams is about $6 \mathrm{~mm}$ and the shutter speed of the camera is uniformly 1 second.

Visualized flow fields are shown in Figs. 14 to 16 . The figures are the flow field on the $Y-Z$ plane at $X=2.0, X$ $-Z$ plane at $Y=0.0$ and $X-Y$ planes at $Z=0.25,0.75$, respectively. Vortex formation and its dynamic motions are clearly observed. The second picture of Fig. 14 shows the recurrent appearence of a large pair of vortces, and Fig. 15 shows the shear flow layer beneath the free-surface, the vortices generated on the top of the mountain ridge and the rising flow which is periodically induced at the position of the pair of vortices. Also, in the case of the $X-Y$ plane at $Z=0.25$ in Fig. 16, the influence of the bottom-plate is shown in the shedding

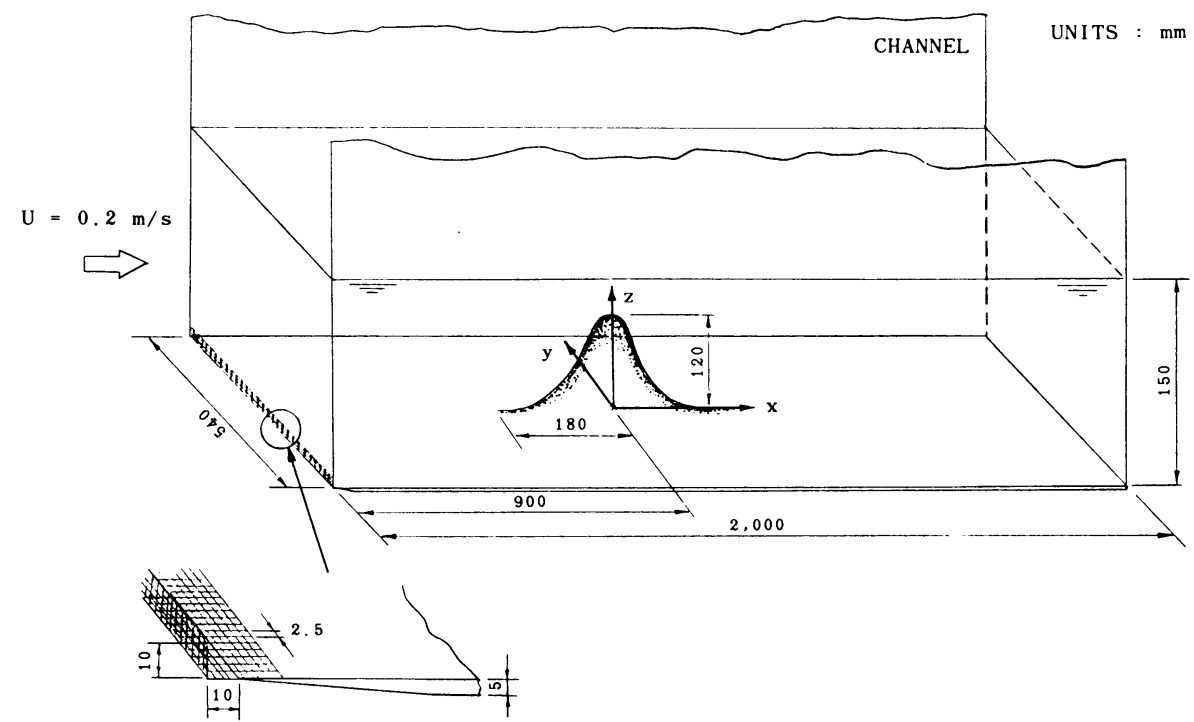

Fig. 13 Coordinate system and experimental set-up for an axisymmetric isolated mountain ridge. 
Numerical and Experimental Study of a Flow past a Submerged Mountain Ridge in Shallow Water 183
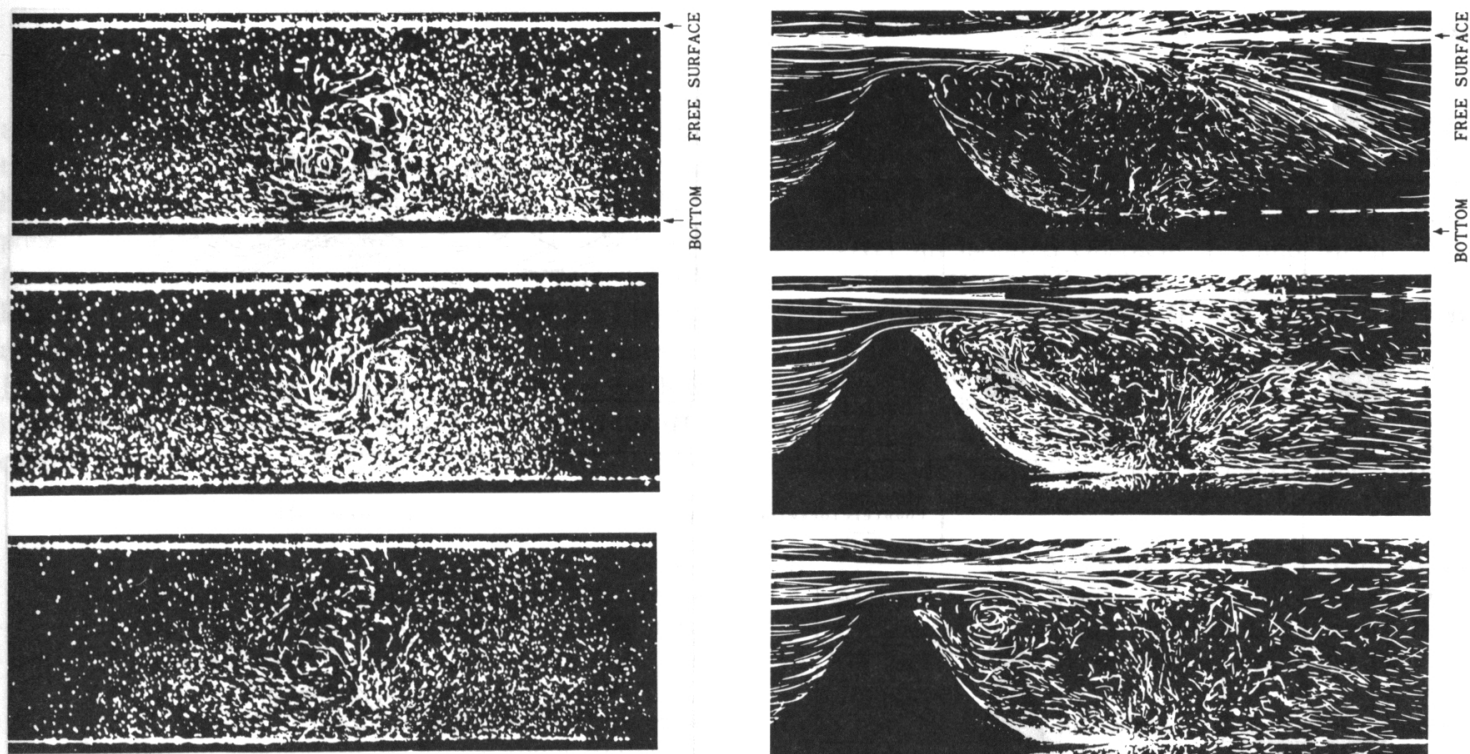

CENTER OF MOUNTAIN RIDGE

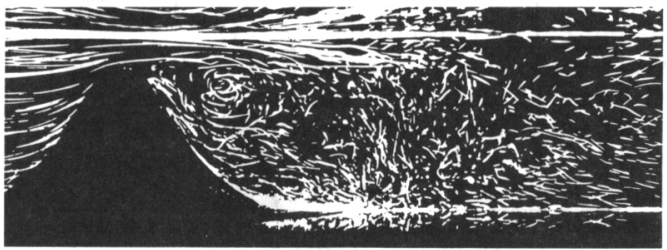

Fig. 14 Visualized flow field on the $Y-Z$ plane of a submerged mountain ridge at $X=2.0$.

Fig. 15 Visualized flow field on the $X-Z$ plane of a submerged mountain ridge at $Y=0.0$.
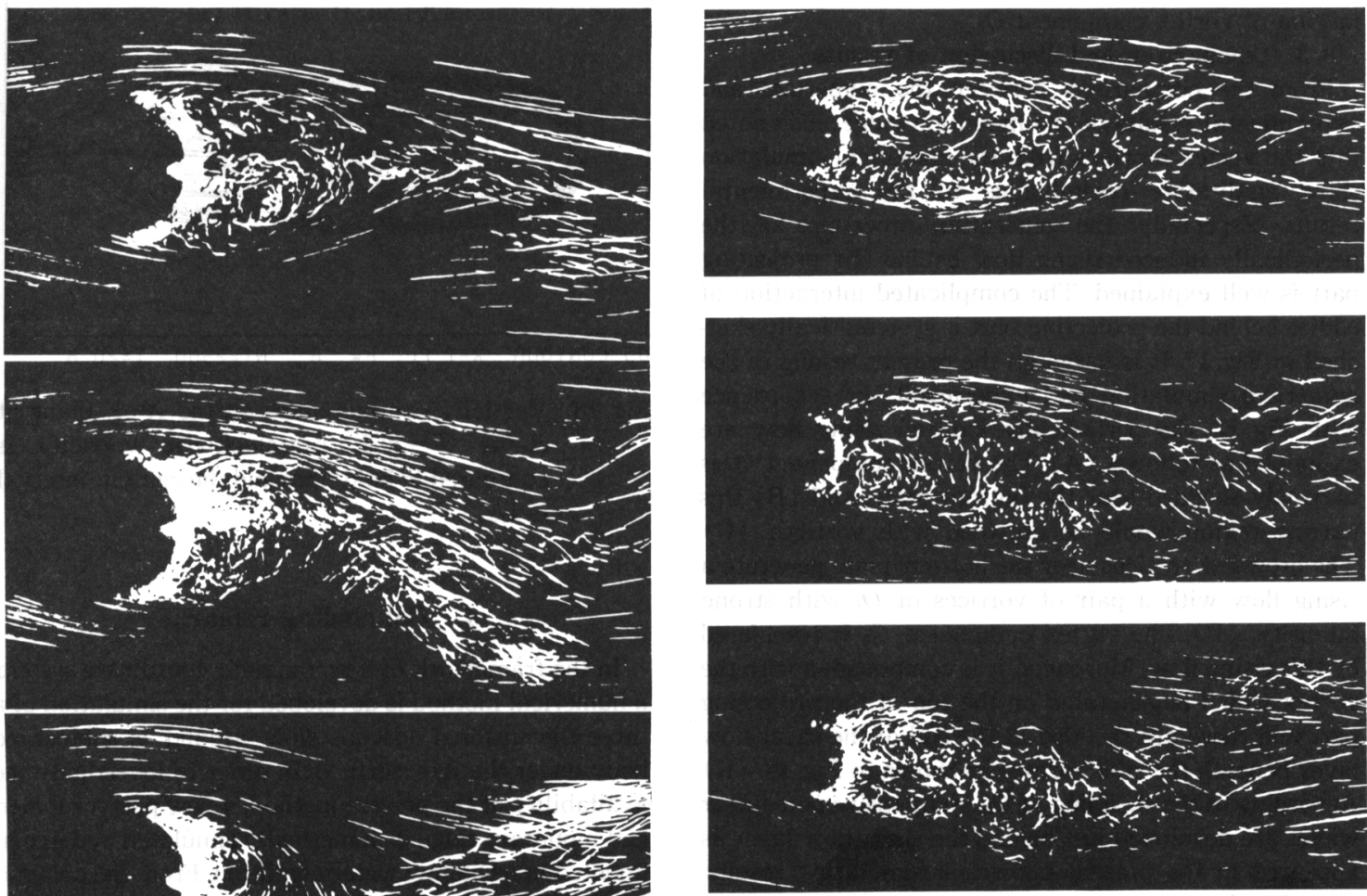

(B) $Z=0.75$

Fig. 16 Visualized flow field on the $X-Y$ planes at $Z=$ 0.25 and $Z=0.75$ 
(A)

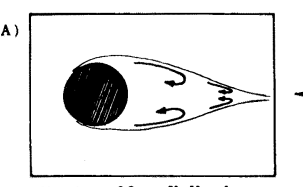

Vortex $03 \quad X-Y$ plane

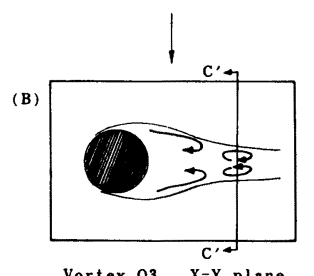

Vortex $03 \quad X-Y$ plane

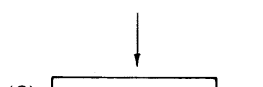

(C)

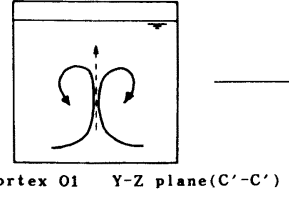

(F)

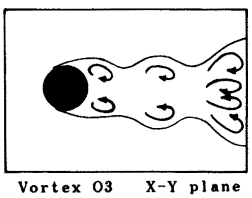

Vortex $03 \quad X-Y$ plane

(E)

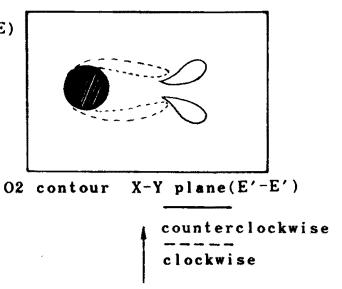

(D)

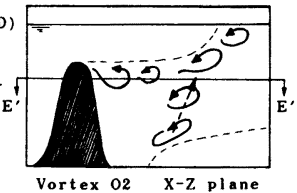

Fig. 17 Sketch of the time cycle of vortex pattern behind a submerged mountain ridge.

motion of vortex component $\mathrm{O}_{3}$.

\section{3 Comparison and discussion of results}

From the comparison between the numerical and experimental results of the mountain ridge, it is noted that the vortex motions from the numerical simulation show qualitative resemblance to the experimental results. Especially, the generation procedure of the periodically induced rising flow behind the projecting part is well explained. The complicated interaction of eddies behind the projecting part is systematically sketched in Fig. 17. It is based on the present results of the numerical simulation and experiment. In accordance with Fig. 17, the periodically induced rising flow are explained as follows. (A) The vortex component $O_{3}$ is narrowly shedded near the bottom-plate, and (B) this narrow region would be crowded with vortices. (C) The crowded vortices near the bottom-plate generate a rising flow with a pair of vortices of $O_{1}$ with strong intensity. (D) The vortex component $O_{2}$ is transfered by this rising flow. Moreover, it is compounded with the vortex which is generated on the top of the projecting part and these are shedded at the end of the shear flow layer beneath the free-surface as shown in Fig. 18. (E) According to the shedding of $O_{2}$, an intensive non-linear waves are developed just behind the shear flow layer as appeared in the present numerical simulation results. (F) Finally, the vortex component $\mathrm{O}_{3}$ is shedded together with the vortex component $O_{2}$, too. This procedure is periodically repeated in the wake flow behind the mountain ridge.
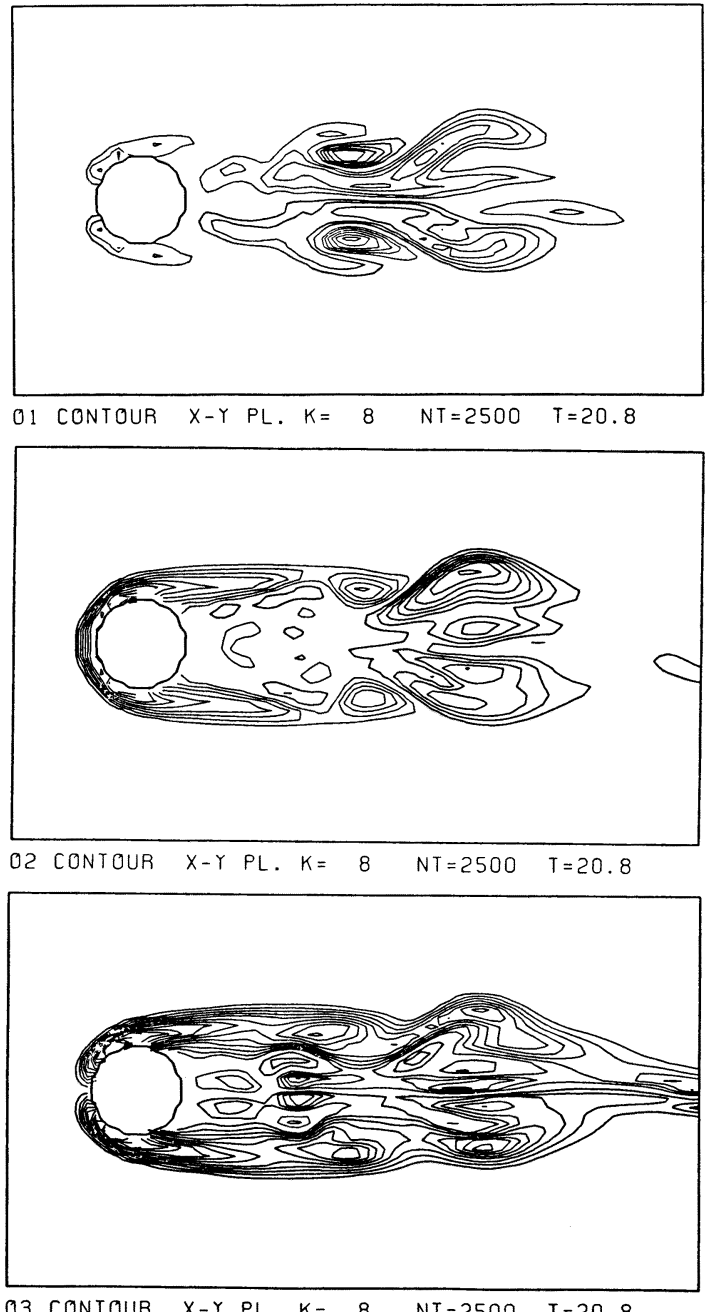

3 CONTOUR $X-Y$ PL. $K=8 \quad N T=2500 \quad T=20.8$

Fig. 18 Vorticity contour maps on the $X-Y$ plane at $K=8, \quad T=20.8$. Anticlockwise vorticity is contoured in bold lines and the contour interval is $1 / s$.

\section{Concluding remarks}

In the framework of a rectangular coordinate system, a numerical method is developed for the simulation of a three-dimensional viscous flow around a submerged body under the free-surface. In order to investigate the availability of the present method, a flow past a submerged mountain ridge is numerically simulated and experimented in a shallow water condition. From the comparison of flow patterns, it is shown that a periodically induced rising flow behind the submerged mountain ridge is generated by the complicated interaction of eddies. The present method is expected to be very useful for the analysis of the complicated three-dimensional viscous flow about an arbitrary object in ocean problems. 


\section{Acknowledgements}

The computations of this research were executed by a computer HITAC M-680 $\mathrm{H}$ in the computer center of the University of Tokyo. The CPU time was about 25 hours for the main computation of this paper. The present work is partly supported by the Grant-in-aid for Cooperative Research of the Japanese Ministry of Education, Science and Culture and also by the LINEC research group organized by shipbuilding companies in Japan. The authors are thankful to Professor $H$. Kajitani for his encouragement, Miss $J$. $-H$. Kim for her careful typewriting.

\section{References}

1) Yamada, Y. and Miyata, H. : A Finite-Difference Method for a Separating Flow Past a Body of Arbitrary Geometry in Rectangular Coordinate Systems, J. Soc. Naval Archit., Jpn, Vol. 167 (June 1990), pp. 17-24.

2) Lee, Y. -G. and Miyata, H. : A Finite-Difference Simulation Method for 2D Flows about Bodies of Arbitrary Configuration, J. Soc. Naval Archit., Jpn, Vol. 167 (June 1990), pp. 1-8.

3) Miyata, H. and Lee, Y. -G.: Vortex Motions about a Horizontal Cylinder in Waves, Ocean Engng, Vol. 17 (1990), No. 3, pp. 279-305.

4) Lee, Y. - G., Miyata, H. and Kajitani, H. : Some
Applications of the TUMMAC Method to 3D Water-wave Problems, J. Soc. Naval Archit., Korea, Vol. 25 (Dec. 1988), No. 4, pp. 13-27.

5) Houghton, D. D. and Kasahara, A.: Nonlinear Shallow Fluid Flow Over an Isolated Ridge, Comm. Pure Appl. Math., Vol. 21 (1968), pp. 1-23.

6) Lamb, V.R. and Britter, R. E.: Shallow Flow Over an Isolated Obstacle, J. Fluid Mech., Vol. 147 (1984), pp. 291-313.

7) Miyata, H., Matsukawa, C. and Kajitani, H.: A Separating Flow Near the Free Surface, Osaka International Colloquium on Ship Viscous Flow, Japan (1985).

8) Cullen, M. J. P. : Implicit Finite Difference Methods for Modelling Discontinuous Atmospheric Flows, J. comput. Phys. 81 (1989), pp. 319348.

9) Lee, Y. - G.: A Finite Difference Simulation Method for the Viscous Flow with Free Surface by Using Rectangular Coordinate Systems, Doctor thesis, the Univ. of Tokyo, september 1989 (in Japanese).

10) Chan, R. K. C. and Street, R. L.: A Computer Study of Finite-Amplitude Water Waves, J. Comput. Phys. 6 (1970), pp. 68-94.

11) Deardorff, J. W. : A Numerical Study of ThreeDimensional Turbulent Channel Flow at Large Reynolds Numbers, J. Fluid Mech., Vol. 41, Part 2 (1970), pp. 453-480. 